\title{
Spatially-variant Strength for Anatomical Priors in PET Reconstruction
}

\author{
Yu-Jung Tsai, Student Member, IEEE, Georg Schramm, Johan Nuyts, Senior Member, IEEE, Sangtae \\ Ahn, Member, IEEE, Charles W. Stearns, Fellow, IEEE, Alexandre Bousse, Simon Arridge and \\ Kris Thielemans, Senior Member, IEEE
}

\begin{abstract}
This study explores the use of a spatially-variant penalty strength, proposed initially for quadratic penalties, in penalized image reconstruction using anatomical information. We have used the recently proposed Parallel Level Sets (PLS) anatomical prior as it has shown promising results in the literature. It was incorporated into the previously proposed preconditioned algorithm (L-BFGS-B-PC) for achieving both good image quality and fast convergence rate. A 2-dimensional (2D) disc phantom with a hot spot at the center and a 3D XCAT thorax phantom with lesions inserted in different slices are used to study how surrounding activity and lesion location affect both the visual appearance and quantitative consistency, respectively. Anatomical information is provided and assumed to be well-aligned with the corresponding activity images. For the XCAT phantom, the inserted lesions are either present or absent in the anatomical images to investigate the influence of the anatomical penalty. The reconstructed images for both phantoms with and without applying the spatially-variant penalty strength are compared. Preliminary results demonstrate that applying the spatially-variant penalization with an anatomical prior can reduce the dependence of local contrast on background activity and lesion location. Further work to explore the potential benefit in clinical imaging is warranted.
\end{abstract}

\section{INTRODUCTION}

$\mathbf{I}$ $\mathrm{N}$ emission tomography (ET), penalized image reconstruction methods are one of the strategies to control noise amplification as iterations increase. Desired properties, such as sharp edges and smoothness in uniform regions, can also be introduced by the incorporated penalty function. However,

Manuscript received November 10, 2017. This work was supported by GE Healthcare, by CCP PET-MR (EPSRC grant EP/M022587/1) and by the National Institute for Health Research, University College London Hospitals Biomedical Research Centre.

Yu-Jung Tsai is with the Institute of Nuclear Medicine, University College London, London NW1 2BU, UK (e-mail: yu-jung.tsai.14@ucl.ac.uk).

Georg Schramm is with the Department of Imaging and Pathology, Katholieke Universiteit Leuven, Leuven 3000, Belgium (e-mail: georg.schramm@uzleuven.be).

Johan Nuyts is with the Department of Imaging and Pathology, Katholieke Universiteit Leuven, Leuven 3000, Belgium (e-mail: johan.nuyts@uzleuven.be).

Sangtae Ahn is with GE Global Research, Niskayuna, NY 12309 USA (e-mail: ahns@ge.com).

Charles W. Stearns is with MICT Engineering, GE Healthcare, Waukesha, WI 53188 USA (e-mail: Charles.Stearns@med.ge.com).

Alexandre Bousse is with the Institute of Nuclear Medicine, University College London, London NW1 2BU, UK (e-mail: a.bousse@ucl.ac.uk).

Simon Arridge is with the Department of Computer Science, University College London, London WC1E 6BT, UK (e-mail: S.Arridge@cs.ucl.ac.uk).

Kris Thielemans is with the Institute of Nuclear Medicine, University College London, London NW1 2BU, UK (e-mail: k.thielemans@ucl.ac.uk). tuning the weight between the likelihood term and the penalty term of the objective function is difficult and the optimal value can vary for different datasets. In addition, the use of the penalty function also amplifies non-uniform image resolution, as the effective penalty strength at every voxel is determined by the activity level of itself and its surroundings [1]. This makes visual comparison and quantitative analysis for regions having different activity levels or at different locations difficult even for lesions in the same image. The issue has been studied extensively for quadratic priors but literature is limited for edge preserving [2] or anatomical priors.

The aim of this study is to explore the use of a spatiallyvariant penalization scheme, proposed initially for quadratic penalties [1] and further generalized for non-quadratic penalties [2], in penalized image reconstruction using an anatomical penalty function. The expected benefit is to obtain similar local contrast, independent of surrounding activity and location in the field-of-view (FOV).

\section{METHOD}

\section{A. Objective function}

Penalized image reconstruction is performed by optimizing a function $\Phi$ consisting of 2 parts, the unpenalized likelihood $L$ and the penalty function $R$, with a constant parameter $\beta$ which controls the strength of the penalty.

\section{B. Spatially-variant penalty strength}

In addition to using $\beta$ to regulate the global weight of the penalty term, a spatially-variant penalization map $\kappa$ has been proposed for quadratic penalties to achieve uniform resolution across regions with different activity levels [1]:

$$
R(\boldsymbol{f})=\frac{1}{2} \sum_{k} \sum_{j \in \mathcal{N}_{k}} \omega_{j k} \kappa_{j} \kappa_{k} \varphi\left(f_{j}-f_{k}\right)
$$

where $\mathcal{N}$ is a given neighborhood and $\omega_{j k}$ indicates the weight between voxel $j$ and its neighboring voxel $k$. The latter is chosen to be translationally invariant over the image. In [1], the map $\boldsymbol{\kappa}$ is defined via a diagonal approximation of the Hessian of the log-likelihood which needs a backprojection of the inverse of the measured data. In this study, we approximated $\kappa$ by a precomputable matrix $\hat{\kappa}$ :

$$
\left.\hat{\boldsymbol{\kappa}}=\sqrt{\operatorname{diag}\left\{\boldsymbol{A}^{t} \operatorname{diag}\left\{\frac{\boldsymbol{g}}{\left(\boldsymbol{A} \boldsymbol{f}_{0}+\boldsymbol{n}\right)^{2}}\right\} \boldsymbol{A 1}\right.}\right\}
$$


where $\boldsymbol{f}_{0}$ is the initial image, $\boldsymbol{g}$ is the measured data, $\boldsymbol{n}$ is the expected background events and $\boldsymbol{A}$ is the system matrix where every element $A_{i j}$ denotes the probability that an emission from voxel $j$ is detected by bin $i$. Note that $\hat{\kappa} \approx \kappa$ in [1] when the initial image is close to the solution.

\section{Anatomical penalty function}

As Parallel Level Sets (PLS) has shown promising results [3], it was chosen for exploring the use of $\hat{\boldsymbol{\kappa}}$ with anatomical penalties in this study. Given the PET image $f$ and the anatomical image $\boldsymbol{v}$, the penalty function is defined by:

$$
\varphi(\boldsymbol{f})=\sqrt{\alpha^{2}+|\nabla \boldsymbol{f}|^{2}-\langle\nabla \boldsymbol{f}, \boldsymbol{\xi}\rangle^{2}}, \boldsymbol{\xi}:=\frac{\nabla \boldsymbol{v}}{\sqrt{|\nabla \boldsymbol{v}|^{2}+\eta^{2}}}
$$

where $\nabla$ is a gradient operator and $\alpha$ and $\eta$ are parameters that modulate the strength of the edge preserving property of the function [3]. Note that PLS is equivalent to smoothed total variation (TV) where no anatomical information is present.

\section{D. $L-B F G S-B-P C$}

PLS was incorporated into a fast convergent reconstruction algorithm, L-BFGS-B-PC, which was previously proposed by our group [4], [5] for achieving both good image quality and fast convergence rate. The algorithm performed L-BFGS-B in a transformed coordinate system to circumvent its potential slow convergence and sensitivity to global scale factors. The transformation was achieved as follows:

$$
\boldsymbol{f}^{\prime}=\boldsymbol{D} \boldsymbol{f}, \boldsymbol{D}=\sqrt{\hat{\boldsymbol{\kappa}}^{2}+\epsilon}
$$

where $\epsilon$ is a small constant for preventing the division by zero problem $(\epsilon=0.01$ in this study). Note that we used $\hat{\boldsymbol{\kappa}}$ to calculate the transformation matrix as it is also related to a diagonal approximation of the Hessian.

\section{E. Data}

1) $2 D$ disc phantom: To study how the surroundings would affect the quantitative consistency of a region of interest (ROI), a disc phantom with a hot lesion (value $=3$ ) inserted right at the center of a large hot $($ value $=5)$ or cold $($ value $=1$ ) background (BG) was used. The voxel size of the phantom was $2.397 \mathrm{~mm}$ and the diameter of the feature was 21.573 mm. The attenuation map $(\mu)$ with value of $0.096 \mathrm{~cm}^{-1}$ for the feature and $0.172 \mathrm{~cm}^{-1}$ for the $\mathrm{BG}$ was used for providing the anatomical information as well. Note that the feature had the same absolute difference to the BG in both activity and $\mu$ map hence the effect of PLS would be the same for feature in cold and hot BG. The projection data were generated by using STIR [6] projector in 2D acquisition.

2) 3D XCAT thorax phantom: To evaluate the potential benefit of using $\hat{\kappa}$ in a more realistic condition, XCAT phantom in thorax region [7] (voxel value ranged from 0 to 1) with 6 hot lesions inserted in different slices was also used to generate data representing typical FDG scans, see Fig. 2. The voxel size of the phantom was $3.125 \mathrm{~mm}$ and the diameter of each lesion was $9.375 \mathrm{~mm}$. None of the lesions was in the central slice and 2 of them were in the liver. The uptake of the liver was either high (value $=0.8$ ) or low (value $=0.2$ ) to simulate different background levels for lesions in the liver. Each lesion had the same absolute difference to its surrounding in both activity (difference $=0.4$ ) and anatomical images (difference $=20 \mathrm{HU}$ ) hence the same influence of the anatomical prior. As lesions in liver would have similar linear attenuation coefficient to the surrounding, our simulations roughly correspond to using CT with injected contrast to provide anatomical information. The phantom was forward projected into sinograms corresponding to data from the GE Discovery STE in 3D acquisition.

Physical effects, such as attenuation and system blurring (with FWHM $=5.2 \mathrm{~mm}$ in tangential and radial directions and $5.7 \mathrm{~mm}$ in trans-axial direction) were considered for both phantoms. For analysis purpose (see Section II-G for more information), we also generated data using the same phantoms but with no feature or lesion.

\section{F. Reconstruction}

L-BFGS-B-PC initialized by 1 full iteration of OSEM with 35 subsets was used for image reconstruction. For each dataset, 2 reconstructions ( 1 with the spatially-variant $\hat{\kappa}$ calculated by (2) and one with a constant penalty strength) were made. The map $\hat{\boldsymbol{\kappa}}$ was normalized to its center such that lesions at the center would be penalized by the same strength. 2 more reconstructions (1 with lesions present in anatomical image and 1 without) were made for the XCAT phantom to investigate the influence of the penalty function when anatomical information about the lesions was present (PLS) or absent (smoothed TV). The parameter set $(\eta, \alpha)$ in PLS was chosen according to the scale of the anatomical images. It was $\left(0.01 \mathrm{~cm}^{-1}, 1\right)$ for the disc phantom and $(5 \mathrm{HU}, 0.1)$ for the XCAT phantom, respectively. The global penalty strength $\beta$ was fixed at 10 for all reconstructions.

\section{G. Evaluation}

The local contrast evaluation was conducted in terms of visual comparison and a contrast recovery estimate $C R$. The merit was defined as:

$$
\begin{aligned}
& C R=\frac{\operatorname{mean}\left(\text { Diff }_{\mathrm{ROI}}\right)}{\text { mean }\left(\text { Diff }_{\mathrm{ROI}}^{\text {true }}\right)}, \\
& \operatorname{Diff}=\left|\operatorname{recon}\left(\boldsymbol{g}+\boldsymbol{g}_{l}\right)-\operatorname{recon}(\boldsymbol{g})\right| .
\end{aligned}
$$

where Diff represents the difference image obtained by subtracting the reconstructed image with lesion(s) (recon $\left.\left(\boldsymbol{g}+\boldsymbol{g}_{l}\right)\right)$ from that without lesion(s) $(\operatorname{recon}(\boldsymbol{g}))$ and $\boldsymbol{g}_{l}$ is the forward projection of the lesion(s). The ROIs were drawn in the center of the feature or lesion with size of $9 \times 9$ voxels for the disc phantom and $3 \times 3$ voxels for the XCAT phantom, respectively.

\section{RESULTS}

Fig. 1 shows images reconstructed with and without using $\hat{\kappa}$ for the disc phantom having either high or low BG uptake. It is hard to delineate the feature from high BG for images reconstructed without using $\hat{\kappa}$ (Fig. 1, first row). However, the benefit of using $\hat{\boldsymbol{\kappa}}$ to preserve local contrast is less significant for lesion in low activity BG (Fig. 1, second row). In terms of 
TABLE I: $C R$ values of the feature of the disc phantom.

\begin{tabular}{ccc}
\hline & BG Hot & BG Cold \\
\hline without $\hat{\boldsymbol{\kappa}}$ & 0.33 & 0.46 \\
\hline with $\hat{\boldsymbol{\kappa}}$ & 0.53 & 0.55 \\
\hline
\end{tabular}
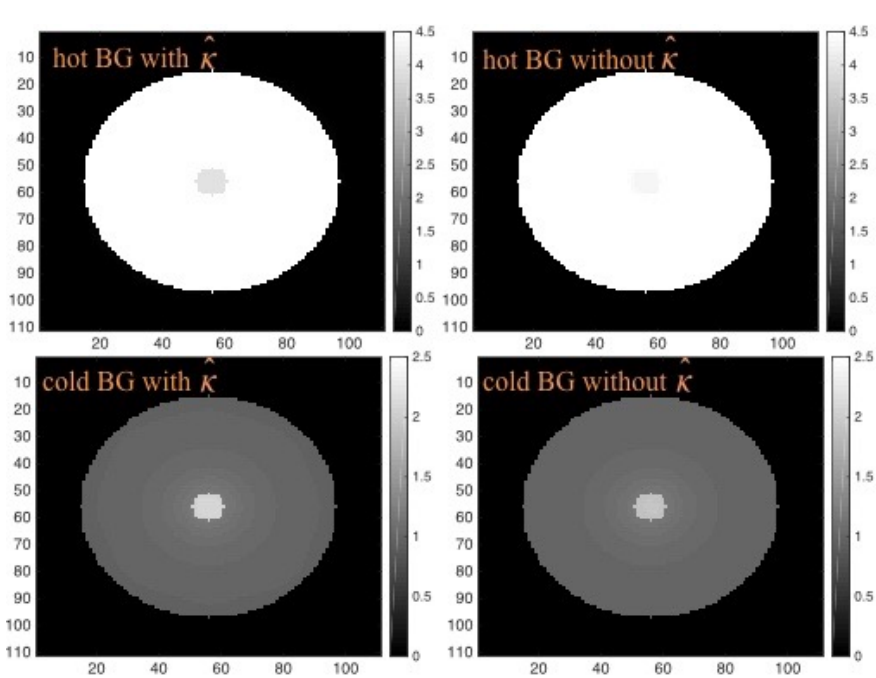

Fig. 1: Converged images reconstructed with and without using $\hat{\kappa}$ for the disc phantom with either high or low BG uptake.

quantitative consistency, the difference between lesion in cold and hot BG is smaller when $\hat{\boldsymbol{\kappa}}$ is applied (Table I). Comparing Fig. 2 to 3 , a relatively uniform visual contrast for lesions at different locations is observed in reconstructions using $\hat{\kappa}$. When a constant penalty strength is applied (Fig. 2), the lesion near end slices is invisible (indicated by purple arrows) if anatomical information is also absent. Although not shown, similar results were obtained for data simulated with low liver uptake. $C R$ values for each lesion of the XCAT phantom under various data simulation and reconstruction conditions are represented using bar charts in Fig. 4. Consistent with the visual comparison based on Fig. 2 and 3, the variance of the local contrast in locations is reduced when $\hat{\kappa}$ is used. However, the influence of surrounding activity is not obvious for the XCAT phantom as lesion 1 and 2 show similar contrast recovery when the activity level of their surroundings (liver) is changed, regardless of $\hat{\boldsymbol{\kappa}}$ is applied or not. Note that the local contrast is significantly increased when anatomical information about the lesions is available (Fig. 2, 3 and 4).

\section{CONCLUSION}

Based on the results for the disc phantom, the influence of surroundings on quantitative consistency can be reduced by applying the spatially-variant penalty strength $\hat{\kappa}$ (Fig. 1 and Table I). The scheme can also be used for improving the uniformity of local contrast across the field-of-view (FOV) (Fig. 2 and 3). However, the dependence of local contrast on surrounding activity was not obvious for the XCAT phantom (Fig. 4). This implies that the local contrast for FDG studies is mainly affected by location (i.e. sensitivity). Future work would include exploring potential benefits of using the spatially-variant penalization scheme on patient data.

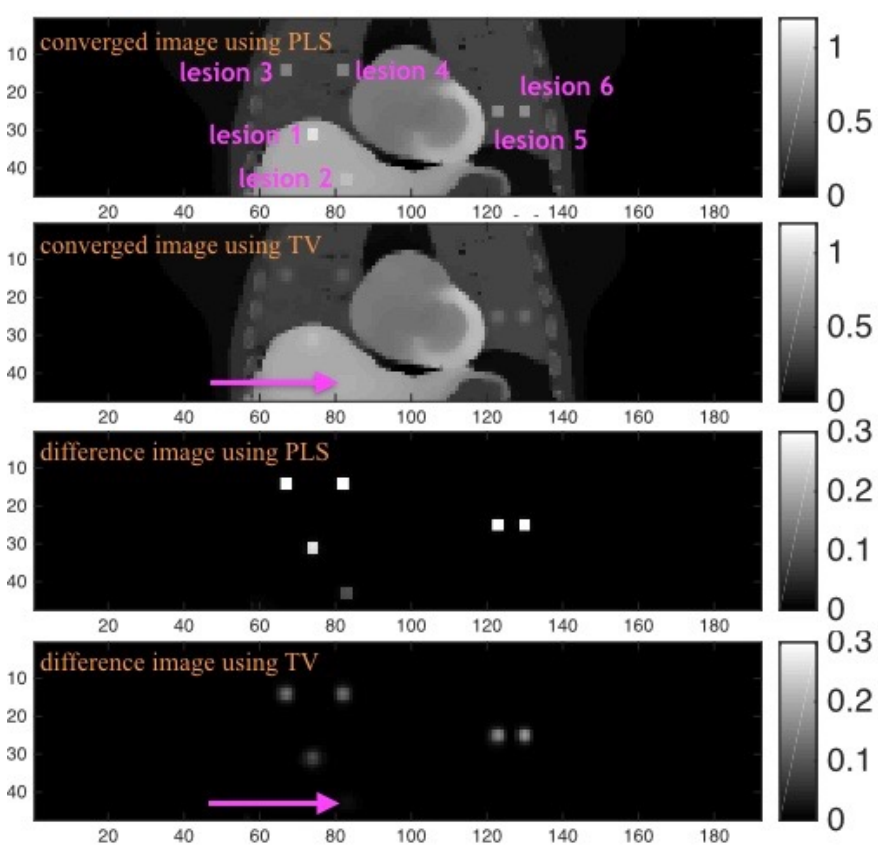

Fig. 2: A coronal view of the converged images reconstructed without using $\hat{\kappa}$ for cases of high liver uptake. The anatomical information about the lesions is either present or absent. The corresponding difference images are also provided.
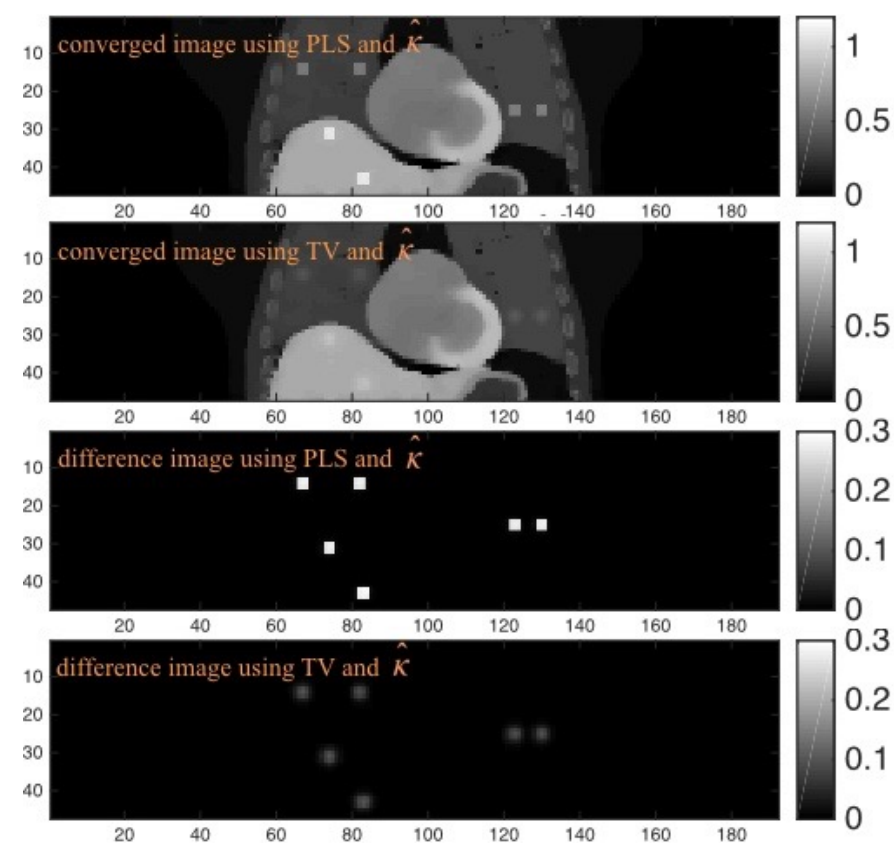

Fig. 3: A coronal view of the converged images reconstructed using $\hat{\boldsymbol{\kappa}}$ for cases of high liver uptake. The anatomical information about the lesions is either present or absent. The corresponding difference images are also provided. 


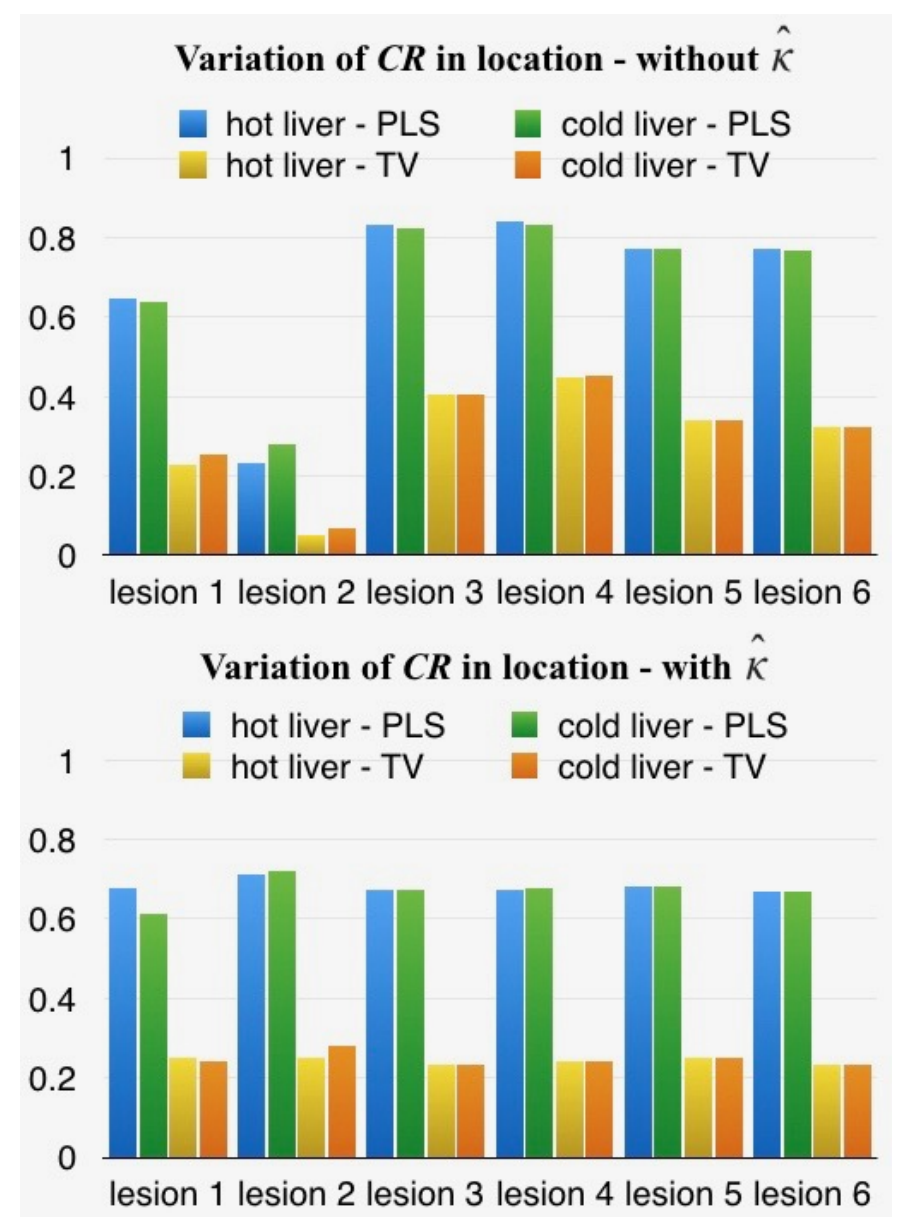

Fig. 4: $C R$ values for each lesion of XCAT phantom under various data simulation and reconstruction conditions.

\section{REFERENCES}

[1] J. A. Fessler and W. L. Rogers, "Spatial Resolution Properties of Penalized-Likelihood Image Reconstruction: Space-Invariant Tomographs," IEEE Trans. Image Processing, vol. 5, no. 9, pp. 1346-58, 1996.

[2] S. Ahn and R. M. Leahy, "Analysis of Resolution and Noise Properties of Nonquadratically Regularized Image Reconstruction Methods for PET," IEEE Trans. Medical Imaging, vol. 27, no. 3, pp. 413-24, 2008.

[3] M. J. Ehrhardt et al., "PET Reconstruction With an Anatomical MRI Prior Using Parallel Level Sets," IEEE Trans. Medical Imaging, vol. 35, no. 9, pp. 2189-99, 2016.

[4] Y.-J. Tsai et al., "Performance Evaluation of MAP Algorithms with Different Penalties, Object Geometries and Noise Levels," IEEE MIC Conf. Proc., 2015.

[5] Y.-J. Tsai et al., "Performance Improvement and Validation of a New MAP Reconstruction Algorithm," IEEE MIC Conf. Proc., 2016.

[6] K. Thielemans et al., "STIR: software for tomographic image reconstruction release 2," Phys. Med. Biol., vol. 57, no. 4, pp. 867-83, 2012.

[7] W. P. Segars, G. Sturgeon, S. Mendonca, J. Grimes, and B. M. W. Tsui, "4D XCAT Phantom for Multimodality Imaging Research," Med. Phys., vol. 37, no. 9, pp. 4902-4915, 2010. 